\title{
Towards Evidence Based Splitting of Organizations
}

\author{
Martin Op 't Land \\ Delft University of Technology, Netherlands \\ Capgemini, P.O. Box 2575, $3500 \mathrm{GN}$ Utrecht, Netherlands
}

\begin{abstract}
The reported research program aims at finding and testing principles for adequately splitting organizations. Using actors from Enterprise Ontology as organization building blocks on one hand and criteria from organization science on the other hand, an expert-meeting was presented the organizationsplitting choices for a part of the Dutch Agency of Public Works and Water Management. The experts could construct their own free-format (gut-feeling) organization choice and they could choose from predefined alternatives, based on the High Internal Cohesion / Low External Coupling criterion and calculated using the min-cut algorithm from graph-theory. The gut-feeling alternative appeared to be close to the (non-trivial) calculated organization alternative, with separation of functions as main reason for difference. Also, business service dependencies appeared to determine organization-splitting far more dominantly than information dependencies.
\end{abstract}

\section{Introduction}

Organizations increasingly [1] split off parts and start cooperating with those parts, for instance in Shared Service Centers or by in- or outsourcing activities. Splitting organizations is believed to make organizations more agile [2] in offering complex products, in participating in complex product-offerings of another party or in timely dropping current products. Such agility has become a business requirement in many lines of business [3], from Defense via car industry to banking industry [4]. Splitting organizations in units with clear customer-supplier-responsibilities, clear competencies and geo-flexibility in operations and ICT also improves its current operations, stimulates entrepreneurship and gives those units a customer-oriented focus, with the potential to broaden the customer base [5]. Through a reduction of 
redundancy in processes and $\mathrm{ICT}$, this results in saving costs, simplifying operations and making those operations more manageable [6].

The question where to split an organization is not an easy one: what criteria and what organizational building blocks should be used? Already in a simple example we can see a complex trade-off in motives of customer intimacy, efficiency, product uniqueness, broadening the product portfolio, cost control and equalizing capacity. And even if the (functional) priorities chosen in those motives are clear, it is not immediately clear how this mix of priorities leads to choices in splitting the enterprise [7]. Dietz [8] proposes to use actors of an Enterprise Ontology according to the Design \& Engineering Methodology for Organizations (DEMO) [9] as organization building blocks. Mulder [10] actually tested the use of actors in organization design, letting the criteria appear bottom-up while discussing the positioning of actors. Op ' $t$ Land [11] adds to this method the test on previously defined situational criteria.

In this paper we extend the method to include testing previously defined general criteria. The extended method is applied in a real-life case study in splitting an organization by an expert-meeting in a Group Decision Support (GDS) room. The participating experts were presented a choice in assigning organizational building blocks, using actors from a DEMO Construction Model. We summarized general criteria from organization science and system theory and tested its use in the actual expert choices. Especially we tested an operationalization of the High Internal Cohesion / Low External Coupling (HICLEC) criterion, as introduced into IS development by Stevens et al [12] and into organizational studies by Karl Weick [13]. We measured the strength of business service dependencies compared with information dependencies. This operationalization took the shape of prepared graph alternatives, using the min-cut algorithm. We found that business service dependencies determined the organization splitting far more than information dependencies. We found also that (non-trivial) calculated organization alternatives appeared to be close to the gut-feeling alternative with separation of functions as the main reason for difference.

The remainder of this paper is structured as follows. Section 2 explains the research design applied, from the level of the research program until the level of an individual case. For each individual case in the program we discern an interventionand a measurement-phase; in this case-study we describe the intervention-phase. Section 3 introduces the actual intervention in the case Rijkswaterstaat- Deltares ${ }^{1}$ : what was its context, how did the models and criteria specific for its situation look like and how was the expert-meeting constructed? Section 4 discusses the results of the intervention: how was the expert-meeting conducted, which of the prepared alternatives were chosen and what were the underlying hypotheses. Finally, section 5 provides the conclusions as well as directions for further research.

Rijkswaterstaat is the Dutch Agency for Public Works and Water Management; this Directorate-General is the implementing organisation of the Ministry of Transport, Public Works and Water Management. Deltares is a Dutch-based international institute for applied research and specialist consultancy in the field of water and the subsurface. For further details, see section 3 . 


\section{Research Design}

This case-study is part of the CIAO-research program [14] "Applying Architecture and Ontology to the Splitting and Allying of Enterprises" [7]. We look for answers to the question "which organization-construction rules lead to adequate splitting of enterprises?". In this section we will first introduce the research design of the research program as a whole, which has the shape of action research. In this action research cycle we then position this individual case study. Next we formulate for this case study the desired exploration and the hypothesis we wanted to test. Finally we explain the structure of the expert meeting used.

\subsection{Position of case study in research program}

The research program "Applying Architecture and Ontology to the Splitting and Allying of Enterprises" aims at finding validated principles and organization construction rules, whose application leads to adequate splitting of enterprises. We define an enterprise as a goal-oriented cooperative of people and means. Splitting and allying enterprises are two sides of the same medal: the moment the work for an enterprise is split over parties, those parties have to ally in order to stay that "goaloriented cooperative of people and means".

Let's now further introduce the basic concepts of the program (see Figure 1). For a specific enterprise $E$ we want to arrive at an adequate splitting proposal (E). Adequate is defined as being compliant with professional principles, enterprise specific principles, situational process requirements and situational result

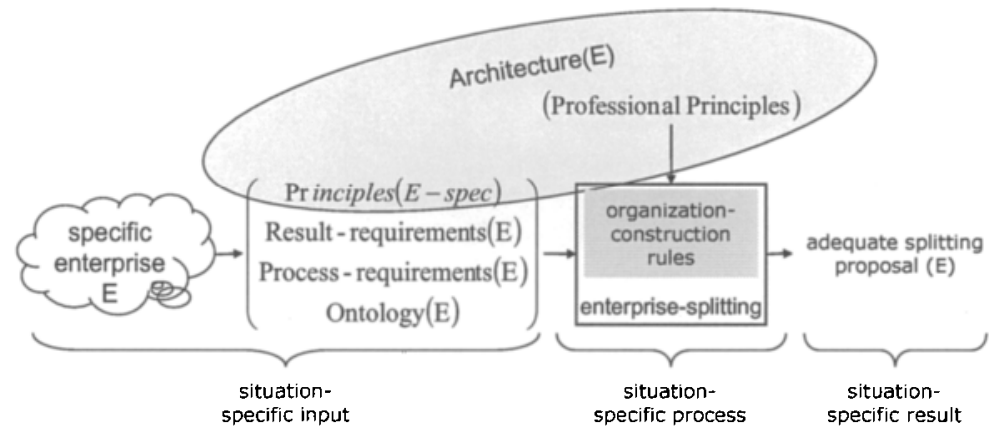

Figure 1. Concepts of Enterprise Splitting

requirements. A professional principle is broadly applicable and not situation specific, e.g. "minimize need for tuning"; it will typically originate from general systems theory and organization sciences. Enterprise specific principles (Principles $(E$-spec $)$ ) are the operationalization of enterprise E's strategic choices and policies, e.g. "re-use before buy before build" or "all employees should be able to work everywhere in our country in the same way". Situational process requirements (Process-requirements(E)) are specific for a specific process or project of splitting, e.g. project costs, timeliness, effectiveness and quality. Situational result requirements (Result-requirements $(E)$ ) are the goals to be reached by splitting the 
enterprise, including the constraints to be complied with. As mentioned in section 1, the goals for splitting of enterprises can be quite diverse and include saving costs (location, people, tax), improving quality (right people with right qualifications in e.g. language, training and experience) and improving agility and flexibility. Constraints will typically originate from the ecosystem of the organization, like from (legal or branch-) supervisors, customers, suppliers and other network partners. Following xAF [15], architecture is understood (1) conceptually as a normative restriction of design freedom and (2) operationally as a consistent and coherent set of design principles that embody general requirements, where these general requirements hold for a class of systems. Therefore the architecture of enterprise $\mathrm{E}$ (Architecture $(E))$ consists of the professional principles and the enterprise-specific principles. By the ontology of the enterprise $\mathrm{E}($ Ontology $(E))$ we understand a model of E's construction that is completely independent of the way in which it is realized and implemented. After Dietz [8], we will use a DEMO Construction Model for such an ontology. Organization-construction rules guide the decisions where to split, e.g. "don't cut the enterprise on a spot with heavy information-exchange, because this will increase the error-rate". In the organization-construction rules the trade-offs are made between all requirements and principles, using the ontology as language of the essence of the enterprise and delivering finally that adequate splitting proposal.

To find the organization-construction rules, which is in the black-box of the enterprise-splitting process, we have to repeatedly execute that splitting process, controlling the in- and output. This fits in the notion of action research, defined by Avison et al [16] as a repeating cycle of intervention, measuring, evaluation and improvement. In action research, the researcher selects or develops new concepts and tools, in our research program organization-construction rules for splitting enterprises, to use it (or let it be used) in new situations. Each case study in the program, including this one, has its own sub problem, method, result and conclusions and therefore also each case has its own research design. As Lee [17] shows, studying single cases can satisfy the standards of the natural science model of scientific research.

Where does this case-study fit in the action research cycle? Dietz [8] proposed to use actors of an Enterprise Ontology according to the Design \& Engineering Methodology for Organizations (DEMO) [9] as organization building blocks. Mulder [10] actually tested the use of actors in organization redesign, letting the criteria appear bottom-up while discussing the positioning of actors. Op 't Land [11] earlier added to this method the test on previously defined situational criteria. In this casestudy we extend the method by a test of previously defined general principles and organization-construction rules. And we apply this method in an intervention, the actual splitting of an organization by an expert-meeting in a Group Decision Support (GDS) room. This case-study should have results on two levels, a case-result and a research result. The intended case-result is an adequate splitting proposal, including an underpinning why that proposal is advisable. This splitting proposal will in turn be the basis for drafting SLA's and for migration planning. The intended researchresult is (1) explorative: which professional principles and organization-constructionrules have been applied and why (2) validating the well-known general construction principle "high internal cohesion, loose external coupling" (HICLEC) as hypothesis. 


\subsection{Using DEMO modelling in the organizational building blocks}

We will now briefly introduce the required concepts of the DEMO Construction Model (CM), using Figure 2. A DEMO Construction Model expresses the coherence (chain/network) of business services, delivered by actors to other actors within a defined scope. E.g. actor A01 executes transaction T01, which delivers a business service to actor A00. Actor A00 is called the initiator and actor A01 the executor of transaction $\mathrm{T} 01$. The execution of transaction $\mathrm{T} 01$ results in a
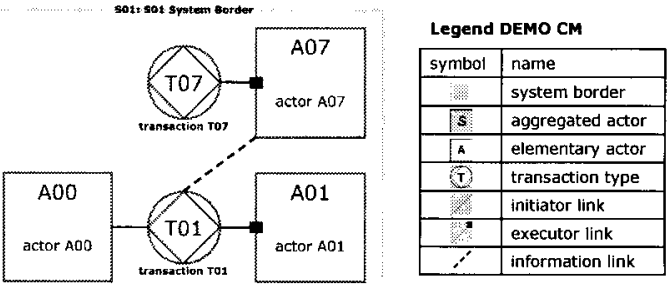

Figure 2. Typical DEMO Construction Model new fact in reality. Another actor A07, for its responsibility in executing transaction T07, needs information about those facts from transaction $\mathrm{T} 01$; this information link between actor A07 and (the fact bank of) transaction T01 is indicated by a dashed line. In the fact bank of T01 we find both the production facts and the coordination facts (like status "requested", "promised", "stated", "accepted") of transaction T01.

In terms of a DEMO CM, making an organization-split is the assigning of actors to separate organizational units. Parnas states [18] that in modularization the modules should be structured in such a way that changes in reality influence the modules in an isolated way, so that modules are islands of stability. In a DEMO CM actors fulfill Parnas" "information hiding" principle, because they have a relatively simple outside interface - a new fact brought about in reality - and potentially hidden complexity on the production process needed to produce that fact.

\subsection{The general criteria for splitting and their application}

Based on the scripts of several case studies [10, 11, 19] and supplemented by organization science and systems theory literature (a/o [20], [21]), Op 't Land [22] summarized organization construction rules and professional principles (see Tables 1 and 2).

Table 1. Professional principles

\begin{tabular}{ll}
\hline nr & Professional principle \\
\hline G01 & better quality of operations \\
G02 & more flexibility in service levels \\
G03 & accelerated operations \\
G04 & accelerated time-to-market \\
G05 & lower operational costs \\
G06 & increased turnover \\
G07 & client centricity \\
G08 & customer ownership \\
G09 & multi-channel offering ability \\
\hline
\end{tabular}


Table 2. Organization-construction rules

\begin{tabular}{ll}
\hline $\mathrm{nr}$ & Organization-construction rule: keep actors together, when ... \\
\hline $\mathrm{C} 01$ & $\ldots$ their mutual interface cannot well be standardized, due to complexity \\
$\mathrm{C} 02$ & $\ldots$ their mutual interface cannot well be standardized, due to frequent change \\
$\mathrm{C} 03$ & $\ldots$ they cannot have a supporting role for other actors \\
$\mathrm{C} 04$ & $\ldots$ they use the same language / culture \\
$\mathrm{C} 05$ & $\ldots$ they operate under the same regulatory, legal and tax-regime \\
$\mathrm{C} 06$ & $\ldots$ those actors more or less work on the same case / deal with the same event \\
$\mathrm{C} 07$ & $\ldots$ the risk to fail (in banking sector: operational risk) of a split is unacceptable high \\
$\mathrm{C} 08$ & $\ldots$ they need comparable competencies \\
$\mathrm{C} 09$ & $\ldots$ a transaction-relationship exists between those actors \\
$\mathrm{C} 10$ & $\ldots$ an information-relationship exists between those actors \\
\hline
\end{tabular}

The general criteria mentioned (G01-G09 and $\mathrm{C} 01-\mathrm{C} 10)$ should be tested in the organization-splitting case study, except for the criteria $\mathrm{C} 09$ and $\mathrm{C} 10$. Indeed, rules $\mathrm{C} 09$ and $\mathrm{C} 10$ can directly be derived from the DEMO CM and therefore didn't need not be tested by the expert-meeting. And as we will see, these two criteria play a special role in the operationalization of the HICLEC-criterion.

Galbraith [21] brings his four organization-design strategies (1. Slack Resources; 2. Self-Contained Tasks; 3. Vertical Information Systems; 4. Lateral Relationships) ultimately back to a trade-off between two variables: either build in / accept slack in cooperation relationships or strengthen the information-relationship. This inspired our curiosity: how important would those cooperation-relationships (freely translated by us to transaction-relationships) be compared with information relationships in deciding about the organizational split? We suppose Galbraith considered information to be an important organization-design variable, because implementing information relationships at that time (1974) was very costly. That could be different today, since the costs of implementing information relations are considerably lower, caused by emerging standards and widely available cheap and reliable ICTinfrastructure.

We therefore like to test the following hypothesis: transaction relations are more important than information relations, when deciding about organizational splits. We did that by calculating several organization-alternatives in which the transaction- and information-relationships got different weights. During the expert-meeting, the experts were asked to choose which calculated organization-alternative they preferred, thus implicitly choosing for a certain weight-ratio between transactionand information-relationships. This procedure is an application of the so-called conjoint analysis, also called multi-attribute compositional models or stated preference analysis [23].

The hypothesis is made operational in the following way.

1. We assigned a weight to each transaction and information relationship in the DEMO CM. A simple example is "all Transaction relationships (T) get the weight 9, all Information relationships (I) get the weight 1"; for short, we code this as $\mathrm{TI}=91$.

2. We interpret those weights as follows (see Figure 2). A high weight of a transaction relationship between actor roles $\mathrm{A} 00$ and $\mathrm{A} 01$ is an indication that those actor roles $\mathrm{A} 00$ and $\mathrm{A} 01$ should stay together in one organization / 
department. A high weight of an information relationship between actor role A07 and the fact bank of transaction T01, we interpret as an indication that the actor roles A07 and (the executing actor role of $\mathrm{T} 01=$ ) A01 should stay together in one organization / department. The underlying assumptions are that (1) information in an information link mostly deals with the production information of the transaction, not its coordination information (2) production information of transaction $\mathrm{T} 01$ is caused by its executor $\mathrm{A} 01$, not by its initiator $\mathrm{A} 00$ (3) coordination information is caused as much by initiator $\mathrm{A} 00$ as by executor $\mathrm{A} 01$.

3. A certain organizational-splitting solution $S$, which splits the organization in two parts - say Org1 and Org2 -, is fully characterized by the collection of actor roles which reside in Org1 and (therefore automatically the remainder of actor roles) in Org2. We consider a relationship between actors to be broken if those actors reside in different organizations.

Now we can define the penalty function $P$ of solution $S, P(S)$, as the sum of weights of broken relationships in Org1 and Org2 in solution $\mathrm{S}$. As an

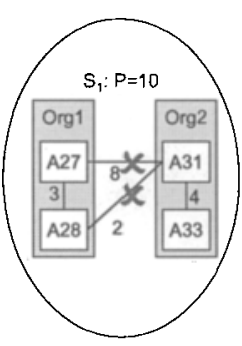

Figure 3. Penalties in Organization Splitting example, see Figure 3: in solution $S_{1}$, the relationships A28-A31 and A27-A31 have been broken, therefore $P\left(S_{1}\right)=2+8=10$; in solution $S_{2}$ only the relationship A31-A33 has been been broken, therefore $P\left(S_{2}\right)=4$.

4. Now we use the HICLEC-criterion: we consider the split better iff the penalty of the splitting-solution is lower. The optimal organization therefore would be the one with the minimum penalty, given that a split has to occur anyhow (the oneorganization alternative doesn't count). This restating of the problem is known in graph-theory as the min-cut problem and a min-cut algorithm exists to solve that [24]. For each weight-distribution (like $\mathrm{Tl}=91, \mathrm{TI}=55$ ), the optimal solution (so with the minimal penalty) could be calculated, also using Gomory-Hu trees [25].

5. Finally it must be possible to state in advance that certain actors should stay with Org1 or Org2 and to enforce that as a boundary constraint for further optimizations.

\subsection{Structure of the expert-meeting}

In the expert-meeting the following steps should be executed.

1. After an explanation of the Construction Model for the area of splitting, as the first step the experts should formulate the gut-feeling alternative. No calculated or other alternative is presented at beforehand, to prevent influencing the experts.

2. Then the gut-feeling alternative should be tested by both situation-specific and general criteria. 
3. After explanation of the calculated organization-alternatives, the experts should express their preference and test the alternatives against the same criteria as the gut-feeling alternative.

4. Finally the experts were asked to answer questions on the way of working.

\section{The intervention: case Rijkswaterstaat - Deltares}

Rijkswaterstaat (RWS), the Directorate-General for Public Works and Water Management is the executive branch of the Ministry of Transport, Public Works and Water Management (V\&W). Under the command of a departmental Minister and State Secretary, it constructs, manages, develops and maintains the Netherlands' main infrastructure networks. RWS aims to prevent flooding, ensure adequate good quality water, ensure safe \& unimpeded movement on roads and waterways and generate reliable information in a user friendly format. RWS has an annual expenditure of approximately $€ 4$ billion, number of staff approximately 10,500, 17 departments and 160 offices in the Netherlands.

From mid-2007, the Netherlands will gain an institute for applied research and specialist consultancy named Deltares (Dlt) [26]. Its goal is to improve the habitability of vulnerable delta areas, contributing to the sustainable management, use and design of low-lying, densely-populated deltas. Deltares wants to be in the international top flight in the field of water and the subsurface. It will use an integrated approach to develop innovative solutions. Deltares brings together Dutch knowledge, experience and specialists in the area of water and the subsurface. The Deltares workforce will be 700 to 800 FTEs in the initial stages. Turnover is projected at $€ 80$ million a year.

Deltares will bring together WL | Delft Hydraulics, GeoDelft, parts of TNO Built Environment and Geosciences and parts of specialist services of RWS. At the time of this case-study, it had to be decided and validated which responsibilities of RWS exactly had to be split off from RWS and added to Deltares.

To guide us in the choice for an area for our case study (which area, which size, when, who to involve) we had to balance the following considerations:

- what is the "right size" of the area for the case study; the good choice here would make it sufficiently interesting and relevant for the RWS-/Dlt-policy makers and at the same time feasible in time;

- political visibility: to what extent and in which phase did we want what attention from what stakeholders to this case study; e.g. the right moment could enable a fruitful discussion between management and Works Council about a major BPO or IT-outsourcing proposal;

- availability of material; during the case study we would have to use existing

DEMO CM-models of Rijkswaterstaat, tested by subject matter experts, and also existing situational principles, process requirements and result requirements.

Waterquantity has been chosen as area of our case-study. This RWS-area is responsible for the hydrological and morphological state of the Dutch national waters. This covers a wide range of activities, varying from a/o modelling hydrology 
and morphology, measuring and reporting water heights, controlling dikes, operating sluices/locks, via the Storm Surge Warning Service to integral consulting on all this.

For the area Waterquantity, a DEMO Construction Model validated by subject matter experts was available. The model emerged from an application consolidation project, in which it was used to structure the current application portfolio, seeking for rationalization-opportunities. The model contained 43 (elementary) actors, 59 transactions and 69 information links.

As boundary constraints for graph-construction RWS-experts in advance chose:

- RWS-BED: all operations of construction works (like sluices, locks and storm surge barriers) should remain with RWS;

- DLT-MOD: all modelling of hydrology and morphology of national waters should go to Deltares.

We chose quite distributed values as parameters for the graph-construction, namely $\mathrm{Tl}=91, \mathrm{Tl}=55$ and $\mathrm{TI}=19$. This delivered three calculated splittingalternatives, named by us $\alpha$ (small RWS), $\beta$ (intermediate) and $\gamma$ (small Deltares). Alternative $\alpha$ (small RWS) only consisted of RWS-BED and alternative $\gamma$ (small Deltares) only consisted of DLT-MOD. Alternative $\beta$ brings all modelling, all consulting services and the SLA-management for hydrology and morphology to Deltares. Both the alternatives $\alpha$ and $\gamma$ are trivial, because they simply reflect the boundary constraint. The alternatives $\alpha$ and $\gamma$ appear as min-cut alternatives for all values of $\mathrm{TI}(19,55$ and 91$)$. Alternative $\beta$ only appears for $\mathrm{TI}=91$, as second choice (penalty $\mathrm{P}=11$ ); the first choices for $\mathrm{TI}=91$ were $\alpha$ and $\gamma$ (both $\mathrm{P}=4$ ). The participants of the expert-meeting were asked to express and underpin their preference for organization-alternative $\alpha, \beta$ or $\gamma$, of course without knowing the value of the TI-parameters underlying those alternatives.

RWS introduced 9 strategic principles and 16 business principles, Deltares introduced 5 principles. Two examples of situation-specific criteria are:

- DR01 = better focus of RWS on networkmanagement (its core-business);

- DR05 = Deltares should be an authoritative knowledge-institution in Europe.

For the expert-meeting we invited \pm 20 persons, representing 4 groups:

1. subject matter experts, like RWS-management and business staff;

2. subject matter and ontology experts from Enterprise Architecture RWS (EAR);

3. ontology-experts and

4. organization scientists.

This variation in expertise should enable us to perform several analyses like:

- to what extent is it possible to propose meaningful organization-alternatives, only possessing ontology-knowledge;

- how much do the evaluations of subject-matter experts differ, depending if they have ontology knowledge or not;

- to what extent do organization scicentists share the opinion of the group - they don't share the ontology-view or the subject matter knowledge, but they have experience with organization design, so with the underlying professional principles and organization-construction rules.

To enable these analyses we used a Group Decision Support Room, in which all argumentation, comments and scores could be systematically collected and subdivided by group. Also it should enable us to direct the facilitation of discussions. 
The duration of the expert-meeting had to be limited to 4 hours, in which a complete scoring of all actors and organization-alternatives on all (42) criteria was not considered feasible. Therefore in the meeting-planning steps were added to select the top 7 from the 42 criteria and to select 8 more or less representative actors.

\section{Results of the intervention}

On January 29, 2007 08:30-12:30, the expert-meeting on splitting RWS-parts concerning Waterquantity to Deltares has been held, supported by the GDS-system MeetingWorks, which collected all contributions of participants electronically.

The longlist of (42) criteria had been sent before the meeting to the experts to comment on that; comments were received neither before nor during the workshop and no new criteria appeared. In the step to select the shortlist (top-7) criteria from the professional principles and organization-construction rules only $\mathrm{C} 07$ (keep actors together when ... the risk to fail (in banking sector: operational risk) of a split is unacceptable high) got selected; all other shortlist-criteria were situation-specific.

In the testing of the gut-feeling organization-alternative 8 out of actors were selected: 2 for which all agreed to keep the actors at RWS, 2 for which all agreed to move the actors to Deltares and 4 from a middle group on which opinions differed.

Due to time constraints the step "test calculated organization-splits on shortlistcriteria" could not be executed.

In drafting the gut-feeling organization-alternative everyone agreed on the boundary constraints RWS-BED and Dlt-MOD. Most participants agreed that Advice-roles belong to Deltares and that Data-gathering should remain at RWS. On information supply the opinions differed; a small minority positioned suppliers of information and drafters of information strategy in Deltares. Controlfunctions (morphological and hydrological) were mainly placed in RWS, though a small minority put its quality control in Deltares: "don't test your own meat" (separation of functions). Also a new actor role was discovered "establisher required Deltaknowledge".

In the comments given several characteristics of RWS and Dlt emerged, which we consider the basis for additions to enterprise-specific principles. For RWS as catchwords were mentioned control, directing, coordination demand-side, executore of policy and steering, control data. For Deltares as catchwords were mentioned specialized consultancy, execution, knowledge-supplier, trusted advisor, models and model data, specialized statistical analyses, strategic knowledge function, should know state of affair watersystems.

Let's now turn to the choices for the calculated organization-alternative. The calculated organization-alternative $\beta$ was preferred and got report-mark 6.2 with a variance $36 \%$ (see Table 3 ). Because this alternative only appeared with $T I=91$, our hypothesis transaction-relations are more important than information-relations, when deciding about organization-splits could not be falsified. 
Table 3. "Give an over-all report mark (scale 1 to 10 ) to the organization-alternative"

\begin{tabular}{|c|c|c|c|c|}
\hline \multicolumn{3}{|c|}{ Organization-alternative } & \multirow{2}{*}{$\frac{\text { Average rating }}{2.2}$} & \multirow{2}{*}{$\frac{\text { Variance }}{32 \%}$} \\
\hline$\alpha$ & "small RWS" & $(\mathrm{TI}=19,55$ or 91$)$ & & \\
\hline$\beta$ & "intermediate" & $(\mathrm{TI}=91)$ & 6.2 & $36 \%$ \\
\hline$\gamma$ & "small Deltares" & $(\mathrm{TI}=19,55$ or 91$)$ & 5.4 & $55 \%$ \\
\hline
\end{tabular}

How close was the gut-feeling alternative to the calculated organizationalternative $\beta$ ? Four actors were differently positioned, which could be explained mainly by separation of functions. We then recalculated graphs to see if different TIweights could generate the gut-feeling alternative. The answer is no, for only one out of four actors this made a difference; the other three actors could not "switch organization" for any TI-weight.

From the prepared professional principles and organizational construction rules, we noticed the use of two of them. The term "need to know" as in "Deltares needs to know the state of the water systems" we saw as C10 "keep actors together when ... an information-relationship exists between those actors". And sometimes specific expertise was mentioned, which pointed to $\mathrm{C} 08$ "keep actors together when ... they need comparable competences".

Some criteria arose which might have a more general value, e.g. "best fit with purposes of organization", "separation of functions", "keep responsibility with the one who is doing the job" and "establishing information needs always by the demand-side". On "establishing information strategy always by the demand-side" discussion arose; some argued that information strategy deals with how information will be supplied, and subject matter expertise of the supplier should play an important role in that, e.g. in the choice of means for monitoring and data collection.

The participants appreciated the way of working in this expert-meeting. The offering of the calculated ( $\alpha-, \beta-, \gamma-)$ alternatives helped to get more clarity on the motives of the preferred organization-split. Also the contribution of non subject matter experts was valued e.g. their comparison of the RWS-Dlt-relationship with the relationship between an airline and the National Airspace Laboratory (NLR).

The results of the scoring of the gut-feeling alternative were consistent with the scoring of the selected actors on the shortlist-criteria. We further noticed that subject matter experts scored outspokenly and homogeneously (low variance). Also we noticed that the method experts more often abstained from voting, explaining that more subject matter insight was required.

A method-expert suggested to better order the list of criteria, e.g. to add the categories function-/ product-requirements, performance-indicators (like lead time or MTBF) and some organization-construction rules (like technical coherence and failure sensibility).

In preparing the next time workshop the following improvements could be made:

- make the criteria SMART in a small group before the expert-meeting;

- test the DEMO Construction Model in a smaller group, especially on the places where discussions on organization-splits can be expected; for this "borderline" actors, put effort in more precisely defining the (direct object of the) transactionresult; e.g. is A027 Supplier statistics the supplier of standard statistics or of specialized statistics? is A024 SLA Manager information supply serving the demand-side or the supply-side or both? 
During the next time workshop the following improvements could be made:

- start with an explanation on the strategic directions of RWS and Deltares;

- then explain consequences for making an organizational split, e.g. "cooperation and allying over the split remain necessary", "introduce SLA's on the split";

- let the splitting of the organization be done top-down, so first at the level of aggregated actors; where the scores on aggregated actors are not unambiguous, drill down to the level of elementary actors; use the time thus saved for more interaction and discussion;

- the question "should this actor go to Deltares or stay with RWS?" is too binary; indeed an actor role can next to sequentially and concurrently also be fulfilled collectively [8: 125], so introduce the option of collective fulfilment of roles;

- during the workshop let the participants first score the actors with business roles, then the actors with informational roles; then participants will be better aware of their assumptions and score more consistently.

\section{Conclusions and further research}

\subsection{Conclusions on the Jevel of this case}

The half-day expert-meeting was considered productive and effective by the participants. Vagueness in criteria and strategic starting points of Rijkwaterstaat and Deltares were revealed and discussed. The use of actors from the DEMO Construction Model as organizational building blocks - which was new for about $50 \%$ of the experts - was generally clearly understood and appreciated. The discussion got an objective basis, responsibilities and dependencies became clear and also new actors were discovered. The pre-calculated organization-alternatives (graphs) made the discussions on the organization-split more directed. E.g. the roles of RWS and Deltares and the underlying situational principles became articulated more clearly than reached by the gut-feeling exercise.

The (non-trivial) calculated organization-alternative $\beta$ came close to the gutfeeling alternative. The min-cut algorithm delivered 3 organization-alternatives, of which 2 were the trivial ones, namely the smallest ones fulfilling the minimum boundary constraints of actor-roles remaining in an organization. The deviation of the $\beta$-alternative to the gut-feeling alternative could be explained almost fully by the criterion separation of functions.

What can we say about our hypothesis "transaction-relations are more important than information-relations when deciding about organization-splits" (section 2)? The (non-trivial) calculated organization-alternative $\beta$ appeared only when giving transaction-relationships a far higher weight than information-relationships ( $\mathrm{TI}=91$ and $\mathrm{TI}=90)$. As soon as information links were assigned a higher weight ( $\mathrm{Tl} \geq 92)$, then only the trivial organization-alternatives appeared. This case-study therefore was not able to falsify our hypothesis.

No calculation of organization-alternatives with whatever uniform weights for transaction- and information-relationships could exactly produce the gut-feeling alternative. Therefore a completely calculable advise on organization-splitting, based 
on the strength of transaction- and information-relationships, which also is recognized by experts as good, has been proven to be impossible.

On the value and the power of discernment of the general criteria (general principles and construction rules), no conclusion can be drawn from this case-study. Because of time-constraints, the expert-group had to select the criteria which they felt to be most important. From the general criteria only one out of 18 was selected and its score did not noticeably differ from the scores on the other (situation-specific) criteria in positioning an actor on either side of the gut-feeling organization-split.

\subsection{Conclusion on the level of the action research}

Situational Method Engineering [27] is characterized by (1) definition of reusable method chunks by reengineering existing methods and models as well as by capturing new ideas, experience and best practices; (2) engineering of new situationspecific methods by assembling method chunks stored in the repository. From existing method chunks [10] [11] we reused (a) the use of actors from a DEMO Construction Model as organization building blocks (b) the use of a Group Decision Support Mechanism. We added to this in section 2 (c) the use of general principles and construction rules, (d) the operationalizion of the HICLEC-criterion on transaction and information links and (e) the use of the min-cut algorithm from graph theory. Sitation-specific in the method applied is a/o the goal of organizationsplitting, which caused us not to ask for optimal multi-clusters, but to request a binary choice ("stays with organization X" versus "goes to organization $Y$ ").

We have discovered that theoretically underpinned organization-alternatives can be calculated, which look plausible in the real-life situation. That calculated alternative need not be the best; many criteria can play a role, which are not part of the starting point, the DEMO Construction Model. The calculated alternatives, represented in graphs, at least then give the insight which penalties are paid in terms of broken or hampered transaction- or information-relationships. These penalties can then be explicitly weighed against the other criteria. Summarizing: if someone wants to deviate from the calculated alternative, he now will be aware of the penalties of that deviation, which enables him to make conscious trade-offs.

\subsection{Future research directions}

To validate the hypothesis "transaction-relations are more important than information-relations when deciding about organization-splits", replication of this research is necessary with variation in organization types and sectors.

To test the value and the power of discernment of the general criteria (general principles and construction rules), these criteria have to be used again in future casestudies in a way which guarantuees scores on all criteria. At the same time the research design for those case-studies have to stay open for discovering new general criteria.

Another interesting question is what would happen when we apply other criteria and other algorithms to the graphs instead of the min-cut algorithm. E.g. in social networks the criterion of "betweenness" appeared to be successful in predicting the 
structure of communities [28]; could this also be applied to the question of organization-splitting? And now we have also restricted ourselves to a binary choice ("should this actor stay with organization $\mathrm{X}$ or move to organization $\mathrm{Y}$ "), resulting in two clusters. We might broaden the question to an open choice ("what actors have close transaction- / information-relationships"), resulting in multi-clusters, which then could be translated to departments or separate legal entities.

\section{Acknowledgements}

We want to acknowledge Richard Jorissen, acting managing director Rijkswaterstaat / National Institute for Coastal and Marine Management (RWS/RIKZ), and Hero Prins, corporate change-manager Deltares, for their sponsorship of the expert-meeting. Karin Middeljans and Kees Buursink generously made available all relevant materials of the Enterprise Architecture Rijkswaterstaat (EAR) team. And Wim Vree, Hans Mulder, Bart Kusse and Martijn Faay supported us greatly in preparing the expert-meeting and analyzing its results.

\section{References}

1 Hackett, BPO-outlook for finance and accounting 2006-2008 (The Hackett Group, 2006)

2 A. Umar, IT infrastructure to enable next generation enterprises. Information Systems Frontiers 7(3), 217-256 (2005).

3 Thomas L Friedman, The World is Flat: A Brief History of the Twenty-first Century (Farrar, Straus and Giroux, 2005).

4 Martin Op 't Land, Bert Arnold, Ariane Engels, FPS: another way of looking at components and architecture in the financial world. Congress paper for the Dutch National Architecture Congress 2000 (LAC2000). www.serc.nl/lac/LAC-2001/lac-2000/3-realisatie/fps.doc

5 Carolien van Straten, Disaggregating the firm by means of Business Process Outsourcing. Master thesis Erasmus University Rotterdam, 2002. www.strategie-vsb.nl/pdf/8.pdf

6 Lance Travis and Jim Shepherd, Shared Services, Cost Savings, Compliance Relief and Prelude to Outsourcing (AMR-research, 2005).

7 Martin Op 't Land (2006) Applying Architecture and Ontology to the Splitting and Allying of Enterprises; Problem Definition and Research Approach. Proceedings of the OTM Workshops Montpellier France 2006, (R. Meersman, Z. Tari, P. Herrero et al., Eds.), LNCS, Springer Berlin Heidelberg. http://dx.doi.org/10.1007/11915072 46

8 J.L.G. Dietz, Enterprise Ontology - theory and methodology (Springer, 2006).

9 DEMO (=Design \& Engineering Methodology for Organizations)-website, www.demo.nl

10 J.B.F. Mulder, Rapid Enterprise Design (Dissertation Delft University of Technology, 2006).

11 Martin Op 't Land, Bert Arnold, Jan Dietz, Effects of An Architectural Approach to the Implementation of Shared Service Centers. Financecom05 Regensburg, Germany (2005). http:/www.iw.uni-karlsruhe.de/financecom05/contributions/opt-land-paper.pdf

12 W.P. Stevens, G.J. Myers and L.L. Constantine, Structured design. IBM Systems Journal 13(2) pp. 115-139 (1975). 
13 K.E. Weick, Management of Organizational Change Among Loosely Coupled Elements. In: Goodman PS Associates (Eds) Change in Organizations, Jossey-Bass, San Francisco, CA, pp 375-408. (1982)

14 CIAO! program (2004) Research program on Cooperation \& Interoperability Architecture \& Ontology. www.ciao.tudelft.nl.

15 XAF (2003) Extensible Architecture Framework version 1.1 (formal edition); report of the NAF-working group xAF. See http://www.naf.nl/content/bestanden/xaf-1.1 fe.pdf

16 D. Avison, F. Lau, M. Myers, P.A. Nielsen PA, Action research. Communications of the ACM 42:94-97 (1999).

17 Allen S Lee, A Scientific Methodology for MIS Case Studies. MIS Quarterly 13(1) pp. 33-50 (1989).

18 D.L. Parnas, On the Criteria To Be Used in Decomposing Systems into Modules. Communications of the ACM 15(12) (1972).

19 Erwin van der Graaf Architectuurprincipes voor de afbakening van outsourcebare kavels (2006). http:/www.architecture-institute.nl/master-lab/pdf/ErwinVanDerGraaf.pdf.

20 Pierre van Amelsvoort, De moderne sociotechnische benadering - een overzicht van de socio-technische theorie (ST-Groep, Vlijmen, 1999).

21 Jay R Galbraith, Het ontwerpen van complexe organisaties (Samson Uitgeverij Alphen aan den Rijn - Brussel, 1976).

22 Martin Op 't Land, Organization science and systems theory on the splitting of enterprises (unpublished, 2006).

23 Wikipedia on Conjoint Analysis, http://en.wikipedia.org/wiki/Conjoint_analysis

24 L.R. Ford Jr, and D.R. Fulkerson, Maximal Flow Through a Network, Canadian Journal of Mathematics 8:399-404 (1956).

25 R.E. Gomory and T.C. Hu, Multi-Terminal Network Flows. J. SIAM 9 pp. 551-570 (1961)

26 Deltares-website, http://www.deltares.eu.

27 Situational Method Engineering website, http://matis.unige.ch/research/SME

28 Michelle Girvan and M.E.J. Newman, Community structure in social and biological networks. Proc Natl Acad Sci $U$ S A 99(12), pp. 7821-7826 (2002) http://www.santafe.edu/research/publications/workingpapers/01-12-077.pdf 